\title{
Article \\ Effect of Bottom Geometry on the Natural Sloshing Motion of Water inside Tanks: An Experimental Analysis
}

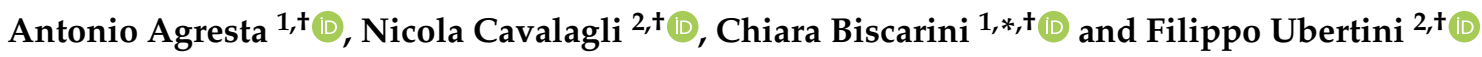 \\ 1 SheC, Sustainable Heritage Conservation Centre, University for Foreigners of Perugia, 06123 Perugia, Italy; \\ antonio.agresta@unistrapg.it \\ 2 Department of Civil and Environmental Engineering, University of Perugia, 06125 Perugia, Italy; \\ nicola.cavalagli@unipg.it (N.C.); filippo.ubertini@unipg.it (F.U.) \\ * Correspondence: chiara.biscarini@unistrapg.it; Tel.: +39-075-574-6677 \\ + These authors contributed equally to this work.
}

Citation: Agresta, A.; Cavalagli, N.; Biscarini, C.; Ubertini, F. Effect of Bottom Geometry on the Natural Sloshing Motion of Water inside Tanks: An Experimental Analysis. Appl. Sci. 2021, 11, 605. https://doi.org/10.3390/ app11020605

Received: 7 December 2020 Accepted: 6 January 2021 Published: 10 January 2021

Publisher's Note: MDPI stays neutral with regard to jurisdictional clai$\mathrm{ms}$ in published maps and institutional affiliations.

Copyright: $\odot 2021$ by the authors. Licensee MDPI, Basel, Switzerland. This article is an open access article distributed under the terms and conditions of the Creative Commons Attribution (CC BY) license (https:// creativecommons.org/licenses/by/ $4.0 /)$.

\begin{abstract}
The present work aims at understanding and modelling some key aspects of the sloshing phenomenon, related to the motion of water inside a container and its effects on the substructure. In particular, the attention is focused on the effects of bottom shapes (flat, sloped and circular) and water depth ratio on the natural sloshing frequencies and damping properties of the inner fluid. To this aim, a series of experimental tests has been carried out on tanks characterised by different bottom shapes installed over a sliding table equipped with a shear load cell for the measurement of the dynamic base shear force. The results are useful for optimising the geometric characteristics of the tank and the fluid mass in order to obtain enhanced energy dissipation performances by exploiting fluid-structure interaction effects.
\end{abstract}

Keywords: tuned sloshing damper; sloshing; beating; passive damping; vibrations; structural control

\section{Introduction}

The aim of this paper is to investigate the dynamic properties of the sloshing phenomenon inside rectangular tanks characterised by different bottom shapes. The interest is to investigate two particular aspects of the fluid-structure interaction, namely the natural sloshing frequency of the fluid inside the tank and its damping feature. The latter is related, among the other features, to the beating phenomenon [1], characterised by the undesired dynamic forces induced on the substructure due to sloshing motion.

The understanding of the aforementioned aspects is important in the framework of the vibration control [2-4], especially in the design process of containers or passive damping devices exploiting fluid motion, since the motions of the container and the liquid are coupled and the resonance phenomena could occur [5-13]. The dynamics of the coupled system generally depend on the container geometry and the amount of the inner liquid. At a first glance, such a problem may be analysed as the case of a bifilar pendulum, even if the physics of such a pendulum is quite different from the sloshing motion inside a container which moves along the horizontal direction. In the case of a container suspended as a bifilar pendulum, gravity directly provides the restoring force, working in concert with, or in opposition, to the hydrodynamic forces, so that both in-phase and antiphase oscillations can occur [14-16]. In the horizontal sloshing case, gravity does not directly affect the motion of the container, being indirectly involved through the liquid motion, whereby the hydrodynamic pressure of the liquid on its walls provides the restoring force. Hence, the sloshing liquid is expected to be out of phase with the oscillating tank, always accumulating in the direction opposite to that of the containers' motion.

In some cases, the interaction between the liquid motion and the substructure could produce significant advantages in the overall dynamics of the coupled system. A classical example is represented by the so called Tuned Sloshing Dampers (TSD) [17-21]. Their 
working mechanism is based on the dynamic response of the liquid motion inside the tank and its interaction with the dynamic input, generally transmitted by the structure on which it is installed [22]. The sloshing forces transferred to the structure are called base shear forces. In real life applications, structural systems are typically induced to move due to external actions, e.g., wind loads or earthquakes and the liquid motion tends to come with a phase shift with respect to the structural motion, depending on the physical properties of the liquid itself, the tank geometries and the structural dynamics properties. This phenomenon results in a dissipation energy by virtue of the energy exchange between the structural system in motion and the device itself. During the dynamic motion, an energy amount is temporarily stored and can be effective in vibration damping when released back to the structure. This energy exchange becomes more effective if a tuning process is properly carried out, so that the sloshing forces are able to reduce the structural vibration and mitigate resonance effects [23].

When excited, the water wave motion quickly develops, but on the contrary, the established wave motion does not vanish immediately after the excitation is over. This results in the so-called beating phenomenon, consisting of long lasting undesired oscillating pulses transmitted to the substructure with an amplitude modulation instead of an exponentially decaying trend. This behaviour of continuous wave motion is often regarded as an adverse effect, for instance within the structural control framework. The beating phenomenon is due to a fraction of the energy absorbed by the device being transferred back to the structure rather than being dissipated within the device itself, which is a result of the complex liquid-tank system [24]. The amplitude modulation is a consequence of the near resonating condition between structural vibration and water sloshing. Damping in liquidtank systems is generated by wave breaking and the impact of liquid on the container walls, as a result of a complex free surface fluid-structure interaction problem [25-27]. For the interested reader, damping and beating phenomena have been widely treated in the literature on mechanical vibrations (e.g., see [28]).

Given the relevance of fluid-structural interaction phenomena in different branches of science and engineering, in recent years several studies have been developed in order to deeply understand the dynamics of sloshing phenomena and model their behaviour in mechanical coupled systems [29-31] with the majority of the applications in the field of civil engineering applications [32]. In this framework, the use of tanks with different bottom shapes has, in fact, revealed interesting properties for technological applications, such as the Tuned Sloshing Dampers (TSDs) not only for the case of sloped bottom shape which represents one of the most studied [33-38] but also for different various geometries [39-43].

Besides the civil engineering applications, significant advantages can be obtained also in different fields by simply modifying the bottom shape of the container. In this way, it is possible to operate on the main properties of the sloshing phenomenon, i.e., natural frequencies, damping ratios, effective mass and stiffness, which regulate the effectiveness of the coupled dynamic systems. Even if it is known that the fluid dynamic response is affected by the bottom geometry of the tank, there are some aspects which are still open and require more investigations. In this work an experimental campaign aimed at investigating the natural sloshing frequencies and the damping properties of a liquid inside a tank characterised by different bottom shapes is carried out and some results compared through analytical solutions available in the literature.

\section{Theoretical Aspects}

The theoretical background of the sloshing motion dynamics has been largely explored in the literature. For the scope of this work, the theoretical aspects related to the analysis of the natural sloshing frequencies for three bottom geometries have been considered, namely the flat, the sloped and the circular shape.

The physics of the sloshing problem is represented for the general case in Figure 1, with $O x y$ being the considered coordinate system. The total mass of the liquid is denoted by $m_{w}$. A motion of the internal liquid generates a variation the free surface, whose 
kinematics is described by $\eta(x, t)$ denoting the vertical movement over the horizontal line. Consequently, fluid horizontal pressures $p_{x}(t)$ and shear actions $\mu_{x}(t)$ over the sides of the tank produce an overall horizontal force, $F_{\text {base }}(t)$, which represents the base shear force developing between the tank and the substructure, obtained by their integral over the dynamic wet perimeter $\mathcal{S}$

$$
F_{\text {base }}(t)=\int_{\mathcal{S}}\left[p_{x}(t)+\mu_{x}(t)\right] \mathrm{d} s
$$

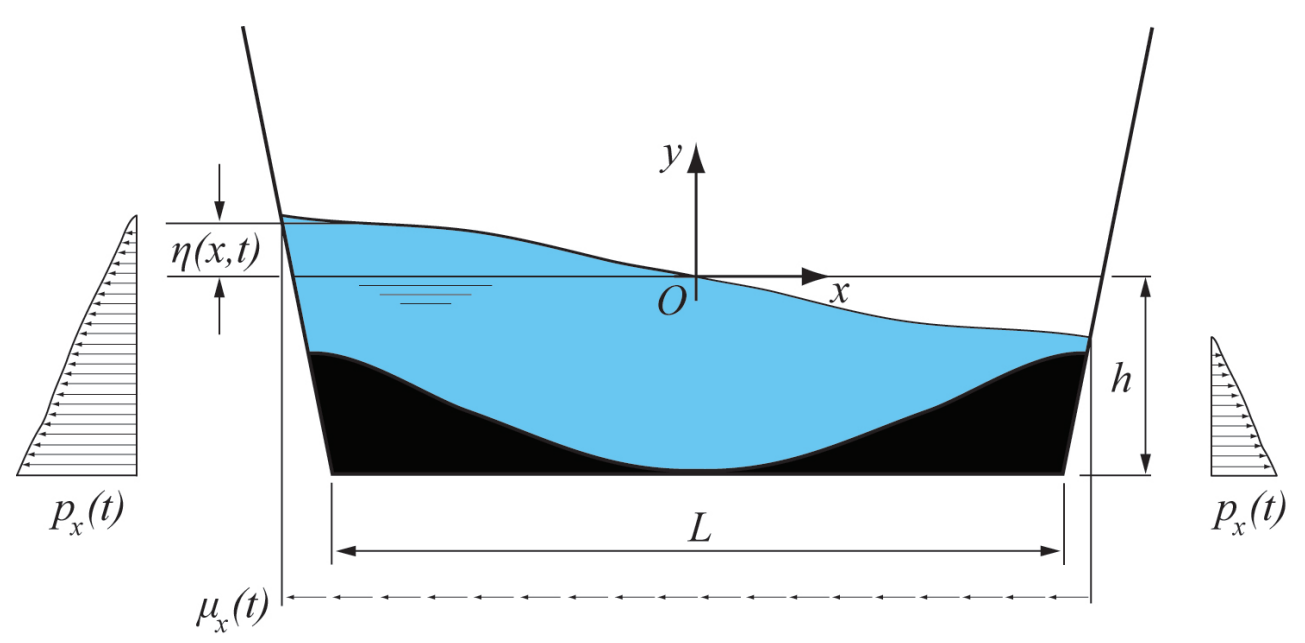

Figure 1. Schematic illustration of the sloshing phenomenon and the horizontal dynamic pressures generating on the sides of a tank having generic bottom shape.

For the flat bottom case, a tank with base length $L$ partially filled with a liquid having depth $h$ has been considered. The value of the ratio $h / L$ approaching zero determines the well-known range of validity of the small amplitude wave theory [44]. Within this range, linear long wave theory can be applied. The classical reference studies, based on the linear wave theory, date back to the end of the nineteenth century, and in particular to the seminal work of Horace Lamb on the mathematical theory of fluid motion [45].

The starting principle underneath the sloshing dynamics theoretical background is the wave motion dynamics described by the classical Laplace equation

$$
\frac{\partial^{2} \phi}{\partial x^{2}}+\frac{\partial^{2} \phi}{\partial y^{2}}=0
$$

Following a standard solving procedure of linear theory, Lamb reports a development through the Bessel harmonics, so that the natural frequency of sloshing for a prismatic tank can be retrieved as a function of tank dimensions and water height [16]

$$
f_{w}^{\mathrm{LT}}=\frac{1}{2 \pi} \sqrt{\frac{\pi g}{L} \tanh \left(\frac{\pi h}{L}\right)}
$$

Deng and Tait [39] derived the expressions of the natural frequency for tanks with different bottom shapes also starting from the linear long wave theory and with the assumption of small free surface fluid response amplitude. In this case, the analogous formulation for the flat bottom geometry results as follows

$$
f_{w}^{\mathrm{LW}}=\frac{1}{2} \sqrt{\frac{g h}{L^{2}}}
$$

It is evident how the dependence from the aspect ratio of volume of fluid inside the tank, $h / L$, represents a crucial parameter. It should be noted that, as stated in [39], the linear long wave theory is valid when $h / L$ approaches zero. In technical applications, 
i.e., related to analysis of sloshing frequencies of water tanks and or TSD, Equation (4) gives accurate values only for $h / L<0.15$. Otherwise, the possible risk is that the values of natural frequency, effective mass, and damping ratio may be overestimated, as this theory assumes that the horizontal component of the velocity is uniform through the liquid depth. In this work, both the Equations (3) and (4) were used for comparison to verify this limitation.

When dealing with different bottom shapes, the contact areas between the liquid and the tank change and the natural sloshing frequency must be differently evaluated.

Concerning the sloped bottom case, the estimation can be obtained through a transformation of the potential in the Laplace equation exploited to extract the free surface height (Figure 2), as proposed by Idir and coauthors [46]. The Laplace Equation (2) given in Cartesian coordinates $O x y$ is still valid, by denoting with flat length the projection of the sloped length to the horizontal axis $L^{\prime}=L / \cos \theta$. The coordinate transfer functions are described by

$$
\begin{aligned}
& x=\sum_{i}^{4} N_{i}(r, s) x_{i} \\
& y=\sum_{i}^{4} N_{i}(r, s) y_{i}
\end{aligned}
$$

where $\left(x_{i}, y_{i}\right)$ are the coordinates of four nodes in the left reference system of Figure 2. The shape functions of the four nodes are as follows

$$
\begin{aligned}
& N_{1}=\left(s / 2 L^{\prime} h\right)\left(2 r-L^{\prime}\right) \\
& N_{2}=\left(s / 2 L^{\prime} h\right)\left(-L^{\prime}-2 r\right) \\
& N_{3}=\left(1 / 2 L^{\prime} h\right)\left(L^{\prime}+2 r\right)(h+s) \\
& N_{4}=\left(1 / 2 L^{\prime} h\right)\left(L^{\prime}-2 r\right)(h+s)
\end{aligned}
$$

(4)

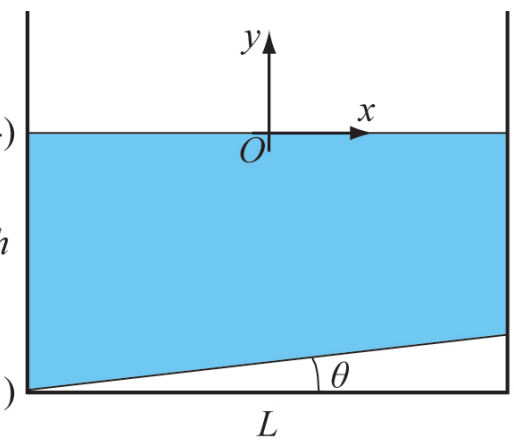

$h-L \cos \theta$

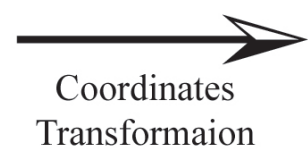

(4)

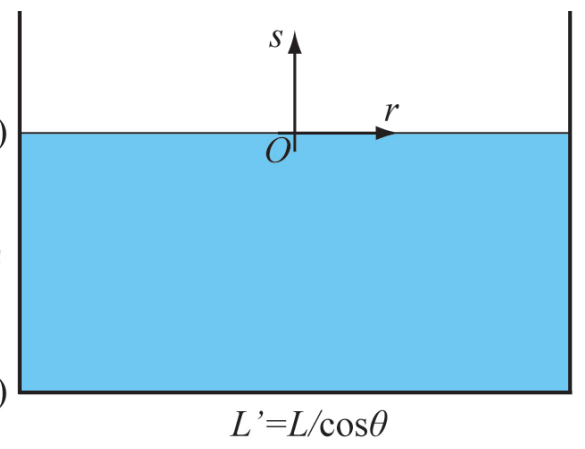

Figure 2. Scheme of the coordinate transformation for the study of the Laplace equation in a sloped bottom tank.

The Laplace Equation (2) can be transformed to the reference coordinate system Ors by differentiation of the potential $\phi$ with respect to coordinate $r$ and $s$ as written in the following equation

$$
\begin{aligned}
& \frac{\partial^{2} \phi}{\partial x^{2}}+\frac{\partial^{2} \phi}{\partial y^{2}}=\frac{\partial^{2} \phi}{\partial r^{2}}\left[\left(\frac{\partial r}{\partial x}\right)^{2}+\left(\frac{\partial r}{\partial y}\right)^{2}\right]+\frac{\partial^{2} \phi}{\partial s^{2}}\left[\left(\frac{\partial s}{\partial x}\right)^{2}+\left(\frac{\partial s}{\partial y}\right)^{2}\right]+ \\
& \ldots+\frac{\partial \phi}{\partial r}\left[\frac{\partial^{2} r}{\partial x^{2}}+\frac{\partial^{2} r}{\partial y^{2}}\right]+\frac{\partial \phi}{\partial s}\left[\frac{\partial^{2} s}{\partial x^{2}}+\frac{\partial^{2} s}{\partial y^{2}}\right]+\frac{\partial^{2} \phi}{\partial s \partial r}\left[2 \frac{\partial s}{\partial x} \frac{\partial r}{\partial x}+2 \frac{\partial r}{\partial y} \frac{\partial s}{\partial y}\right]
\end{aligned}
$$


The right-hand terms of Equation (6) are determined through shape functions differentiation, as follows

$$
\begin{aligned}
& \left(\frac{\partial s}{\partial x}\right)^{2}+\left(\frac{\partial r}{\partial y}\right)^{2}=\left(\frac{1}{\cos \theta}\right)^{2}, \partial^{2} r / \partial x^{2}+\partial^{2} r / \partial y^{2}=0 \\
& \left(\frac{\partial s}{\partial x}\right)^{2}+\left(\frac{\partial s}{\partial y}\right)^{2}=\left(\frac{2 h}{(2 h-L \tan \theta-2 r \sin \theta)}\right)^{2}, \partial^{2} s / \partial x^{2}+\partial^{2} s / \partial y^{2}=0 \\
& 2\left(\frac{\partial s}{\partial x}\right)\left(\frac{\partial r}{\partial x}\right)+2\left(\frac{\partial r}{\partial y}\right)\left(\frac{\partial s}{\partial y}\right)=0
\end{aligned}
$$

By substituting Equations (7)-(9) into Equation (6), the Laplace equation in the reference system $(r, s)$ is obtained

$$
\frac{\partial^{2} \phi}{\partial x^{2}}+\frac{\partial^{2} \phi}{\partial y^{2}}=\left[\frac{1}{\cos ^{2} \theta}\right] \frac{\partial^{2} \phi}{\partial r^{2}}+\left[\frac{2 h}{2 h-L \tan \theta-2 r \sin \theta}\right] \frac{\partial^{2} \phi}{\partial s^{2}}=0
$$

For a small value of $\theta$, the right-hand terms of Equation (10) can be simplified into

$$
\frac{\partial^{2} \phi}{\partial r^{2}}+\frac{\partial^{2} \phi}{\partial s^{2}}=0
$$

Equation (11) is the Laplace equation in the new reference system. Given that it is identical to Equation (2), the natural frequency of the water sloshing motion can be determined directly from Equation (3) by replacing the length with the projection of bottom length to the horizontal axis, which is the contact length between the water and the tank bottom. Generally speaking Equations (2) and (11) can be simplified for a specific case neglecting the inactive components of force and motion, through a proper definition of boundary conditions. Hence, the natural sloshing frequency in the case of the sloped bottom tank is expressed as follows:

$$
f_{w}^{\mathrm{SL}}=\frac{1}{2 \pi} \sqrt{\frac{\pi g}{L^{\prime}} \tanh \left(\frac{\pi h}{L^{\prime}}\right)}
$$

which is formally comparable with Equation (3). The same result, defining a specific wet perimeter, was obtained by Gandarsson in 2001 [33] through the Bessel functions. Nevertheless, as indicated in the literature, the variation of Equation (3) by introducing the wet perimeter is considered valid for small values of $\theta$.

In the reference work of Ibrahim [8], following again what indicated in the seminal text of Lamb [45], a meaningful study concerning the influence of the inclination angle $\theta$ between the lateral walls and the bottom side of the tank was reported.

In particular, with reference to the scheme reported in Figure 3, the solution of the natural angular frequencies for the case of diverging walls at $45^{\circ}$ with the assumption of free surface oscillations independent of the axial coordinate is given

$$
\omega_{m}^{2}=\frac{m}{2 h \alpha}\left[g+\frac{\sigma}{\rho R}\left(\frac{m^{2}}{4 \alpha}-1\right)\right]\left[\frac{1-\kappa^{m / \alpha}}{1+\kappa^{m / \alpha}}\right]
$$

with $m$ being the modal wave number (set to 1 for the first vibrational mode), $g$ the gravitational acceleration constant, $\sigma$ and $\rho$ respectively the liquid surface tension and the liquid density, $R$ the curvature radius of the free surface and $\alpha, h_{1}, k=h_{1} / h_{0}$ being geometrical features as in Figure 3. For the case under analysis, it has been assumed that the curvature of the free surface is small, hence allowing to neglect the second term of the sum inside the square brackets. 


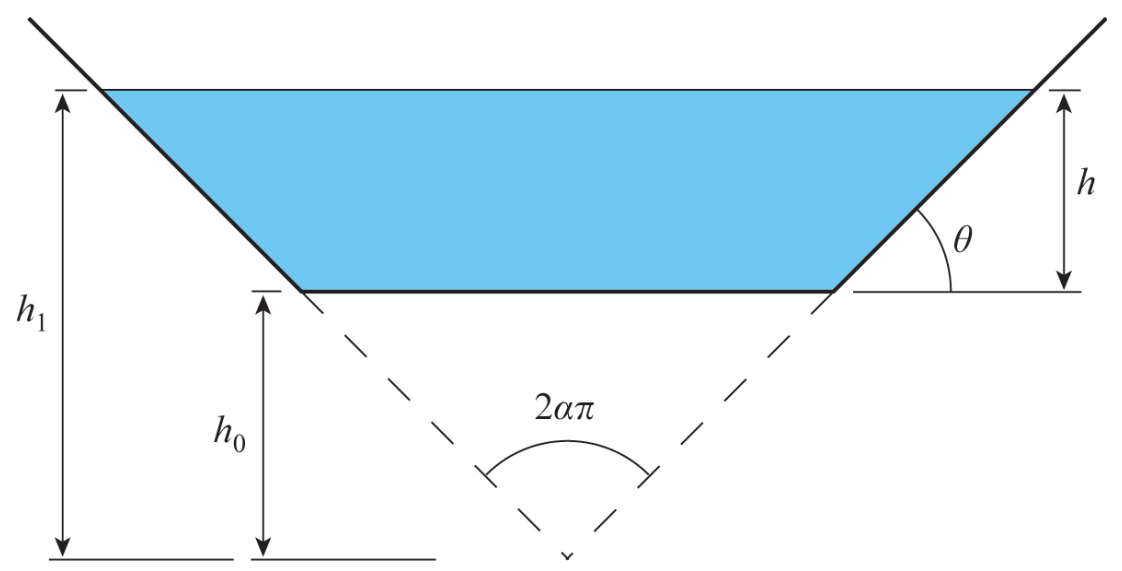

Figure 3. Schematic illustration of a sloped bottom tank with generic angle of embrace of the divergent lateral sides of $2 \alpha \pi$.

Several authors reported studies with explicit analytical solutions for several geometries (triangular bottom, cylindrical, spherical, conical or toroidal containers $[16,20,39,47]$ ), but very few works addressed curved bottom geometries. Deng and Tait [39] proposed an analytical procedure based on the Bessel functions as a solution for the Laplace Equation (2) and derived the expression for both the pulsation and the relative natural frequency in the case of a parabolic bottom tank, respectively, as follows

$$
\begin{gathered}
\omega_{p}^{2}=2 \frac{g h}{L_{0}^{2}} \\
f_{w}^{\mathrm{P}}=\frac{\sqrt{2}}{\pi} \sqrt{\frac{g h}{L^{2}}}
\end{gathered}
$$

where the geometrical parameters $h, L$ are defined as indicated in Figure 4.

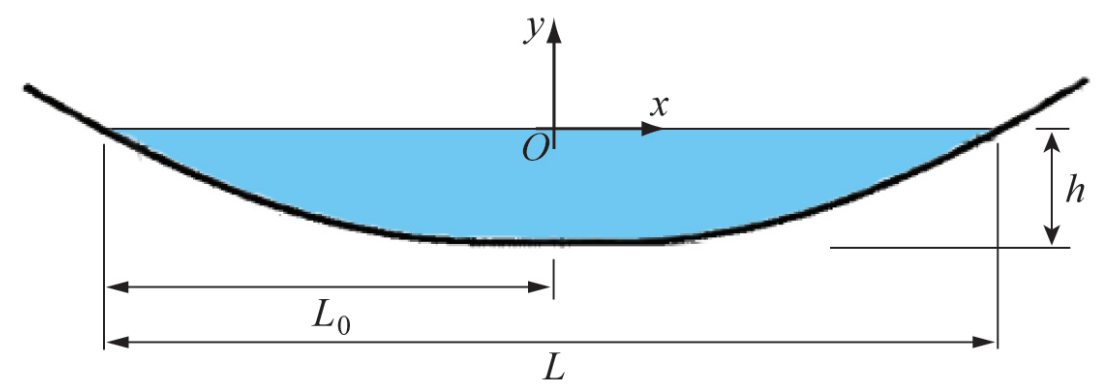

Figure 4. Schematic illustration of a tank with parabolic bottom shape.

\section{Experimental Investigation}

\subsection{Experimental Setup}

The experimental tests were carried out on a tank having a rectangular base with dimensions in plan of $400 \times 200 \mathrm{~mm}$ and then equipped with some apparatuses to change the bottom shape (Figure 5a). The tank is made by Plexiglas plates of $1 \mathrm{~cm}$ thickness to have sufficiently rigid boundaries and at the same time the possibility of observing the movement of the fluid during the sloshing phenomenon. Using the additional 3D printed blocks, whose dimensions are indicated in Figure $5 b$, three different tanks bottom geometries were tested: flat, sloped and circular bottom shapes (Figure $5 c-e)$. For the case of the sloped and circular ones, isosceles wedges of $10 \mathrm{~cm}$ of side and width of $20 \mathrm{~cm}$ corresponding to the width of the tank. The printed blocks were fixed at the base of the tank to prevent relative movements between them. 

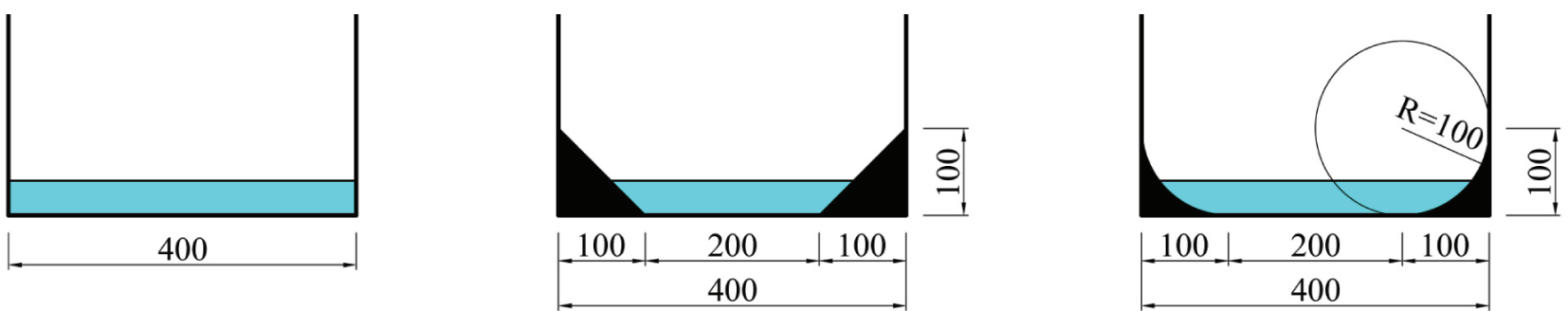

(a)

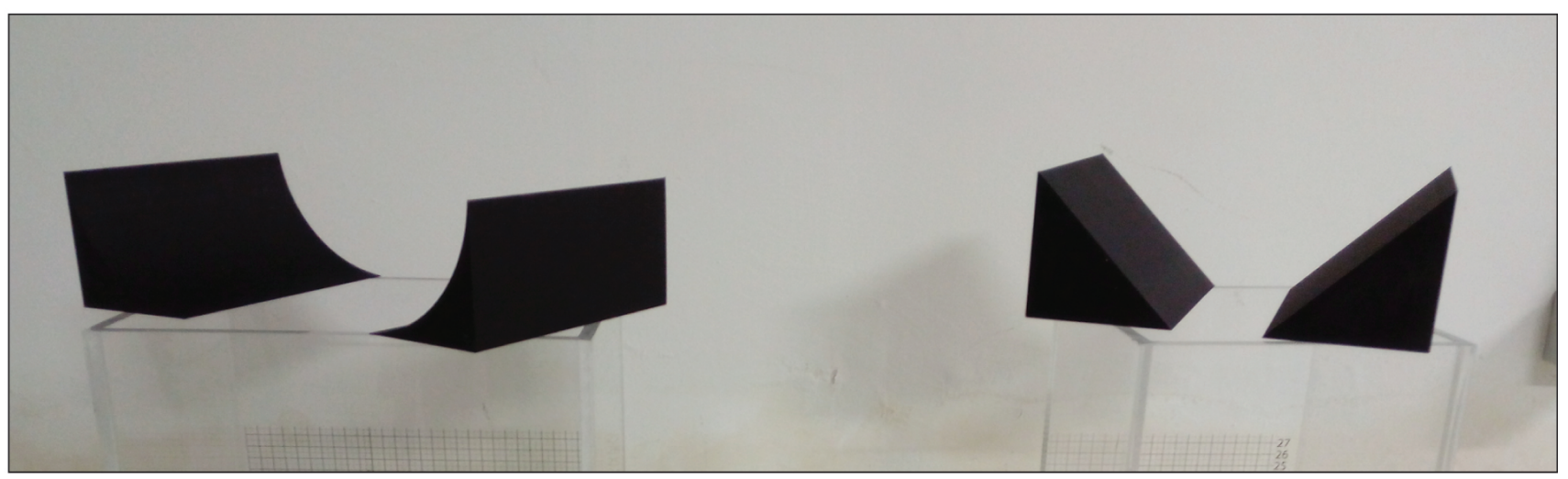

(b)
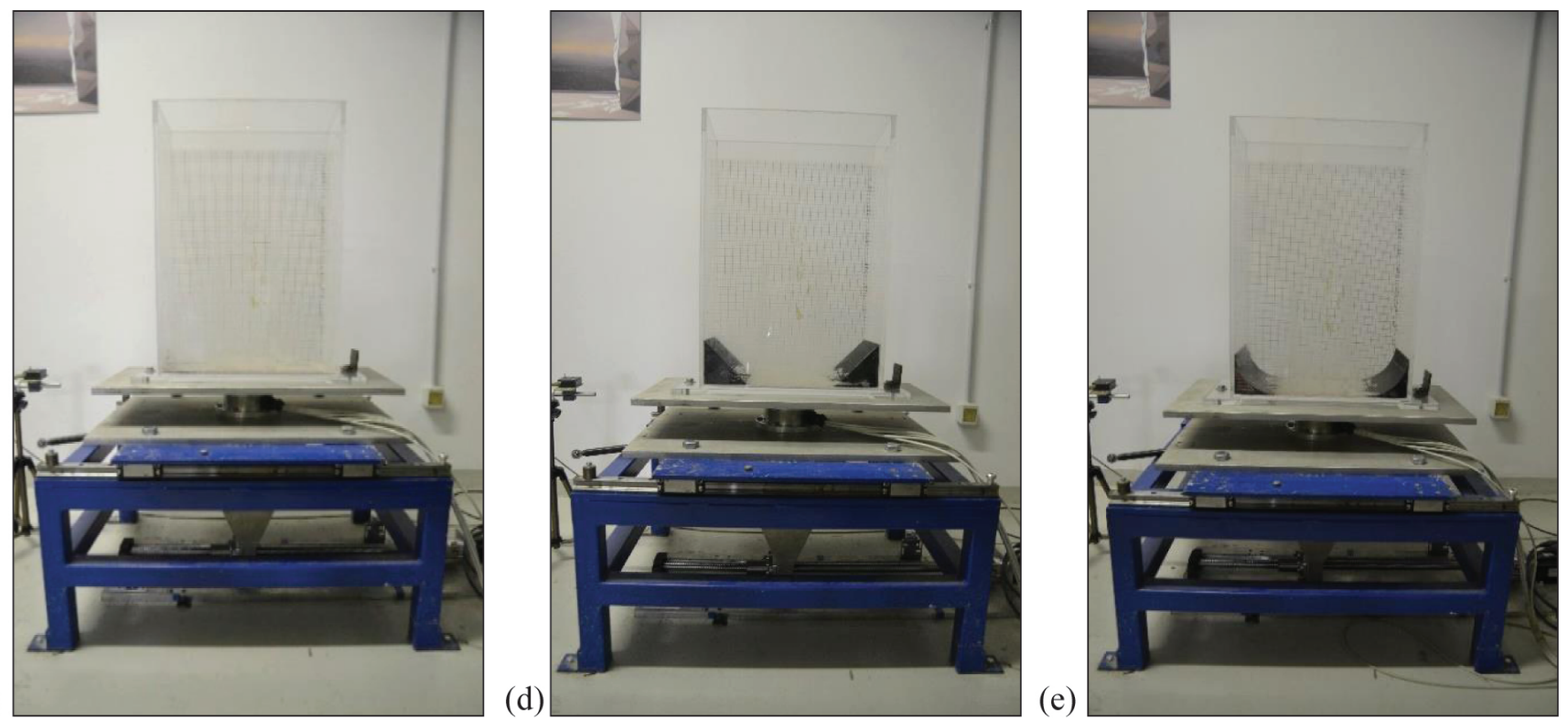

Figure 5. Experimental apparatus. (a) Scheme of the tanks dimensions (measurements in mm). (b) 3D printed blocks used to change the bottom shape. Images of the tanks with flat (c), sloped (d) and circular (e) bottom shape.

The base shear force was measured by a strain gauge dynamic load cell placed between the shaking table $(700 \times 500 \times 20 \mathrm{~mm}$ in dimension) and an aluminium plate, having dimensions of $700 \times 300 \times 15 \mathrm{~mm}$, to which the tank is anchored.

\subsection{Experimental Tests}

The testing matrix consists of 27 different experiments: for each of the 3 different bottom geometries (Figure 5c-e), 9 different levels of water depth, from 4 to $12 \mathrm{~cm}$, with an increasing step of $1 \mathrm{~cm}$, were analysed, allowing to investigate the sensibility of the liquid damping to its volume (mass). The bottom shapes were selected in order to compare and analyse results and relative behaviours.

In the carried experiments, the tanks were excited with an impulsive kinematic input, i.e., a little displacement imposed at the base, to make the inner liquid in free oscillations. In order to analyse the fluid response in a steady-state motion, the initial transient period 
was not considered. The data, sampled for a total duration of $80 \mathrm{~s}$, were acquired with a fairly high sampling frequency equal to $1 \mathrm{kHz}$. A postprocessing procedure was then applied, including a detrend function and a low-pass filter with a cutoff frequency of $5 \mathrm{~Hz}$.

\section{Results and Discussion}

The dynamic data acquired by the load cell were analysed both in frequency and time domains. In Figure 6, as a general example, the base shear force time histories for the three different bottom shapes with a water depth of $7 \mathrm{~cm}$ are illustrated. The experimental data for the base shear force $F_{\text {base }}$ (light grey line of Figure $6 a-c$ ) recall the typical trend of a mass with a single degree of freedom in free vibration in presence of damping. The analytical function which describes the amplitude modulation of a free oscillating damped system (below critical damping) is

$$
F_{\text {base }}(t)=A e^{-\zeta \omega_{w} t}
$$

where $A$ is the amplitude, $\omega_{w}$ is the natural angular frequency and $\zeta$ is the damping.
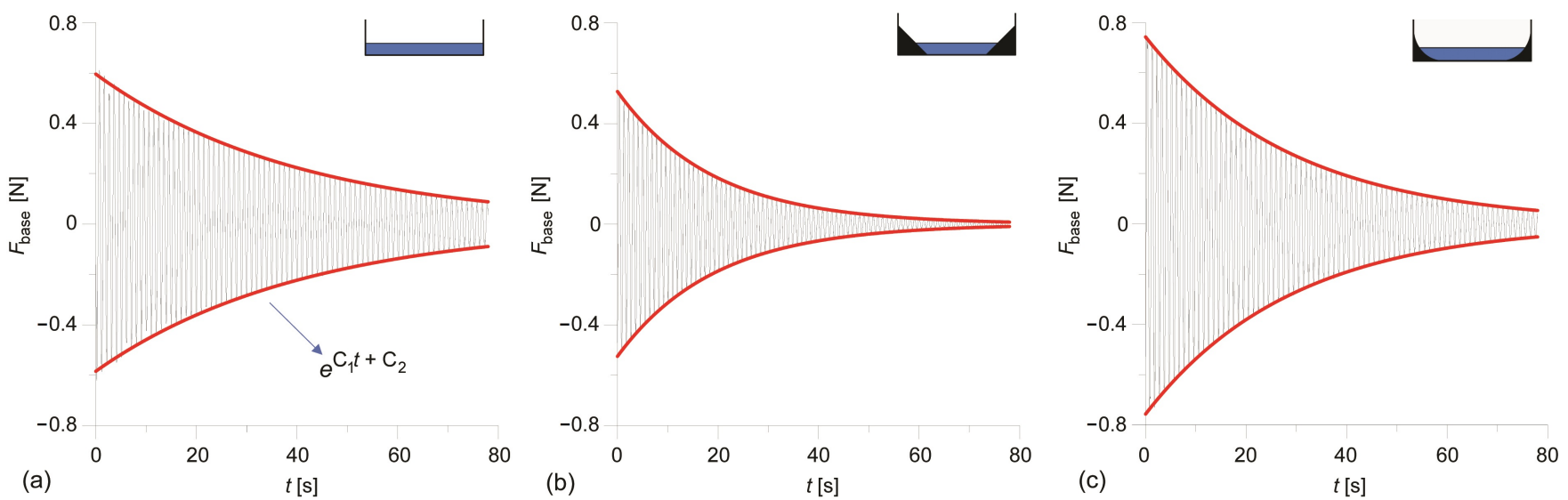

Figure 6. Base force time histories with the calculated enveloping exponential interpolating curves for different bottoming bases at $h=7 \mathrm{~cm}$.

The power spectra were extracted from the dynamic records of the base shear force in order to estimate the natural angular frequency $\omega_{w}$, and then the natural sloshing frequency $f_{w}$, for the different liquid $h / L$ ratios. Table 1 shows a comparison between the natural frequencies for the flat bottom tanks $f_{w}^{\mathrm{EXP}}$ obtained as a result of the experimental campaign. As introduced in Section 2, the experimental results were also compared with the analytical formulations related to the linear theory (Equation (3)) and the linear long wave theory (Equation (4)). In the first column of Table 1, aspect ratios are reported. It is clear how their values, apart from the first two, exceed the limit of validity for the linear theory and yet the error with respect to Lamb's expression is under $2 \%$ for all the different water depths showing a very good agreement. This condition might be explained by the intrinsic nature of the induced sloshing phenomenon under analysis, which might be perfectly compliant with the standard hypothesis of the linear-shallow waters equations.

The impulsive input induced to the tank to make the liquid in free vibrations, together with the geometrical features of the tank, produce a liquid motion that recalls the motion of the iso-potential lines for an irrotational flow, or better put, they generate a set of oscillating waves that closely follow the solution of the system of equations under the potential flow conditions, which is, in fact, the starting point for the analytical solution of Lamb's theory. On the other hand, the comparison with Deng's formulation [39] points out two peculiar aspects. Firstly, relative errors are larger due to the different approach of Deng's work who has employed Lagrange's equations and Morison's formula together with the method of virtual work, an approach that shows a higher sensibility to the geometrical parameters. The second notable aspect is related to the evident increase of the error for water depths higher than $90 \mathrm{~mm}$, (ratio $h / L=0.225$ ) showing, in this case, the actual relaxation of the 
linear-shallow waters hypothesis, as it is even more evident from Figure 7a. This is a consequence of the nonlinear effect of amplitude dispersion that, indeed, resembles that of a hardening spring [33].

Table 1. Comparison between analytical (Lamb and Deng and Tait formulations) and experimental natural sloshing frequencies obtained with the flat bottom tank.

\begin{tabular}{cccccc}
\hline $\mathbf{h} / \mathbf{L}$ & $f_{w}^{\mathrm{LT}}(\mathbf{L a m b})$ & $\begin{array}{c}f_{w}^{\mathrm{LW}} \\
\text { (Deng\&Tait) }\end{array}$ & $f_{\mathcal{w}}^{\mathrm{EXP}}$ & $\begin{array}{c}\text { Err [\%] } \\
(\mathbf{L a m b})\end{array}$ & $\begin{array}{c}\text { Err [\%] } \\
\text { (Deng\&Tait) }\end{array}$ \\
\hline 0.1 & 0.77 & 0.78 & 0.76 & 1.32 & 2.6 \\
0.125 & 0.85 & 0.88 & 0.85 & 0.11 & 2.4 \\
0.15 & 0.93 & 0.96 & 0.92 & 1.09 & 4.7 \\
0.175 & 0.99 & 1.04 & 1.01 & 1.98 & 2.8 \\
0.2 & 1.04 & 1.11 & 1.04 & 0.42 & 6.7 \\
0.225 & 1.09 & 1.17 & 1.10 & 0.91 & 6.9 \\
0.25 & 1.13 & 1.24 & 1.13 & 0.13 & 9.6 \\
0.275 & 1.17 & 1.30 & 1.16 & 0.86 & 11.9 \\
0.3 & 1.20 & 1.36 & 1.19 & 0.84 & 13.9 \\
\hline
\end{tabular}

(a)

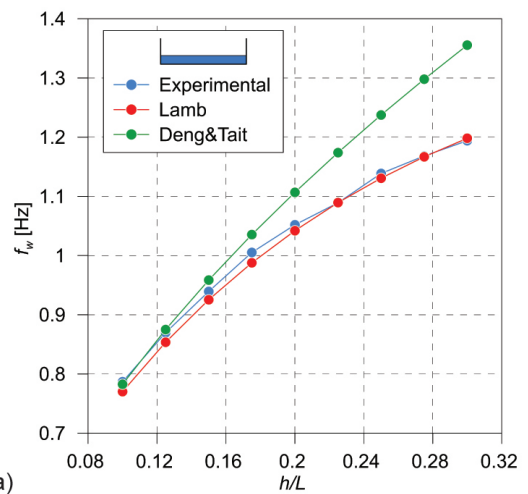

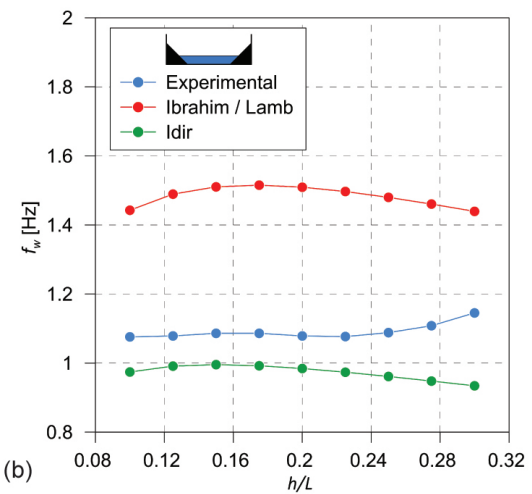

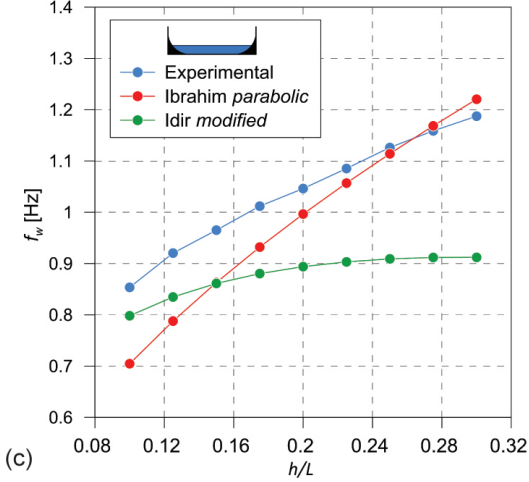

Figure 7. Comparison between analytical and experimental natural sloshing frequencies for flat (a), sloped (b) and circular (c) bottom shape.

Figure 7 gives a comprehensive picture of the differences between experimental data and the different closed analytical approaches, including the sloped and circular bottom geometries, as reported in Figure $7 \mathrm{~b}, \mathrm{c}$, respectively.

The first thing to be highlighted, similarly to the flat case and as expected, is the change of trend for all the three bottom geometries, at values of $h / L$ exceeding the limit of validity of the linear theory. For the flat case (Figure 7a) Lamb's formulation (Equation (3)) seems to be perfectly fitting the data while Deng's modified version (Equation (4)) diverges for $h / L>0.2$, as anticipated above in Table 1 .

For the case of the sloped bottom, the experimental values of $f_{w}$ have been compared to the formulations of Ibrahim [16] represented by Equation (13) and the approach of the wet perimeter proposed by Idir [46] in Equation (12). Both approaches follow a parallel behaviour together with the experimental data up to $h / L=0.2$, showing a maximum between $0.15<h / L>0.175$, but Ibrahim's values (red line) show a fairly big margin of error, probably due to the fact that the empirical equation does not take in consideration the losses from the fluid-structure interactions and frictions. Idir's Equation (12) results showed a fairly good agreement with the experimental data despite the fact that the approach is based on the hypothesis of small angles of the divergent section of the corner wedge.

The circular bottom case comparison is probably the most interesting one since shows two peculiar aspects. On one hand, the experimental $f_{w}$ values have been compared to Ibrahim's (also Lamb's) approach for parabolic bottom shapes [16,45], as in Equation (15), and the result was an overall small error but, as expected, a general trend weakly correlated 
to the obtained data. On the other, Idir's approach (Equation (12)) with a wet perimeter $\left(L^{\prime}\right)$ ad-hoc modified to consider the circular shape of the corner wedges, shows a very good agreement both in terms of relative error and overall compliance of the trend.

In order to estimate the decay function (red line of Figure $6 a-c$ ) characterising the damping properties, an exponential interpolation of the maxima/minima points was computed for the different bottom geometries and for each water depth, by extracting the coefficients $C_{1}$ and $C_{2}$ of the equation

$$
F_{\text {base }}(t)=e^{C_{1} t+C_{2}}
$$

With reference to Equation (16), the damping ratios $\zeta$ were estimated at each level of water depth and for each cases of bottom geometry, taking into account the natural frequencies previously extracted.

Figures $8 \mathrm{a}-\mathrm{c}$ and $9 \mathrm{a}-\mathrm{c}$ summarise the significant evidences of the carried out experiments, as a sort of matrix of the possible responses of the system composed by structure and liquid. More specifically, the beating phenomenon can be related to the slope of the exponential decay functions displayed in Figures 8 and 9 . Figures 8 and 9 clearly highlight that the flat bottom is the least responsive to the height change, meaning that its reaction to the change of water mass is slower if compared to the sloped and the circular bottom shapes. Furthermore, the sloped one, for heights lower than $10 \mathrm{~cm}$, substantially keeps performing with the same timing and shows the same delays with respect to the initial impulse.

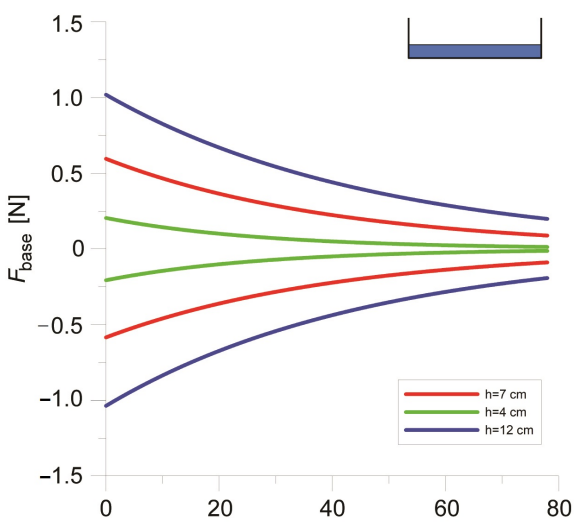

(a)

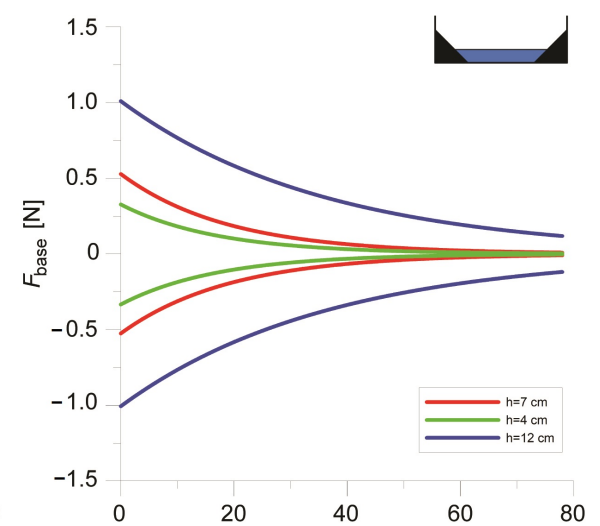

(b) $t$ [s]

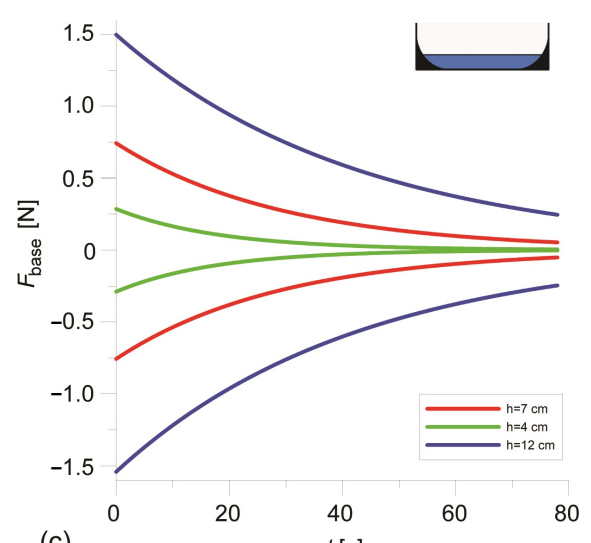

(c)

$t$ [s]

Figure 8. Exponential decay functions interpolated at different water depths for flat (a), sloped (b) and circular (c) bottom shape.

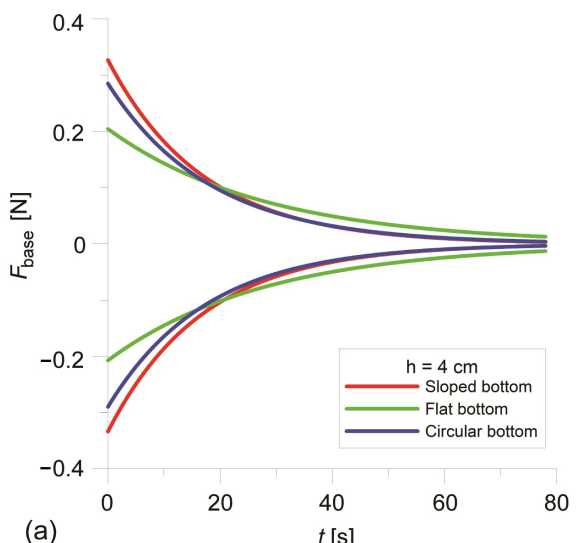

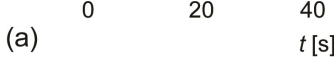

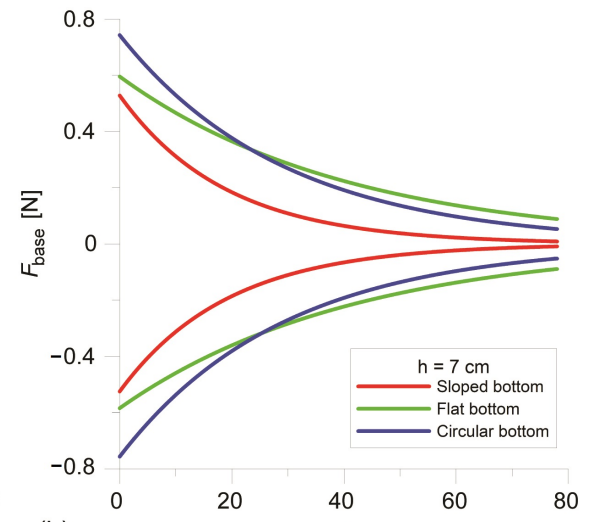

(b)

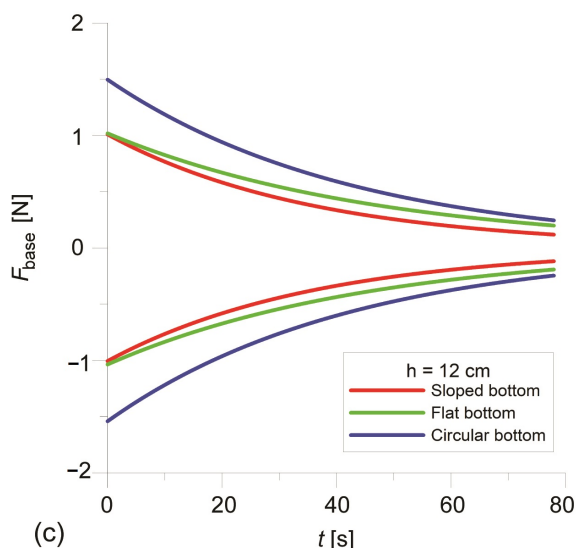

(c)

$t[\mathrm{~s}]$

Figure 9. Comparison of exponential decay functions between the different bottom shapes for water depth of $h=4 \mathrm{~cm}(\mathbf{a})$, $h=7 \mathrm{~cm} \mathrm{(b)} \mathrm{and} h=12 \mathrm{~cm} \mathrm{(c).}$ 
An overall comparison is depicted in Figures 10 and 11, where the trends of only the experimental natural frequencies and damping ratios, with respect to the different water $h / L$ values are represented, respectively.

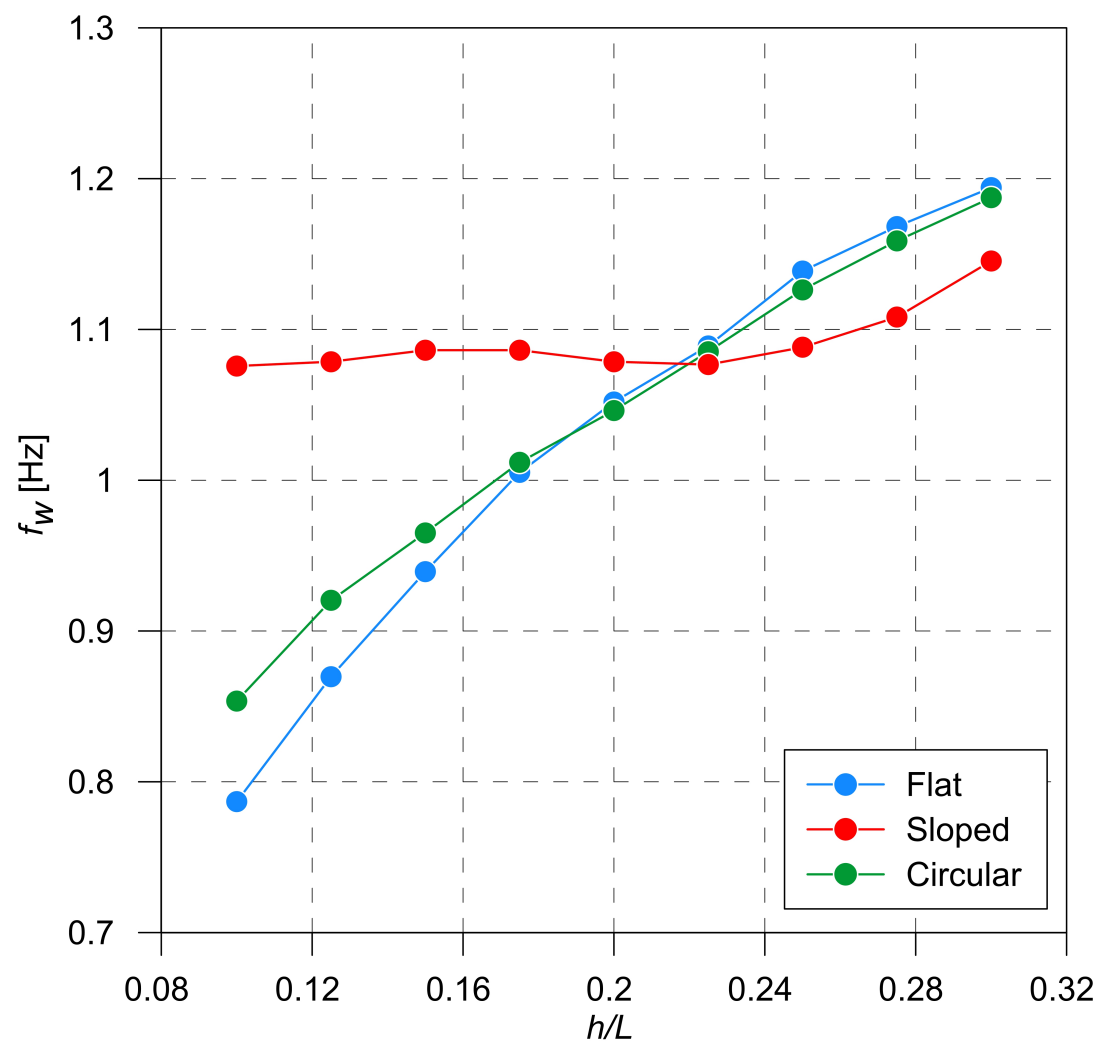

Figure 10. Natural frequency values vs. water depth ratio for different bottom geometries.

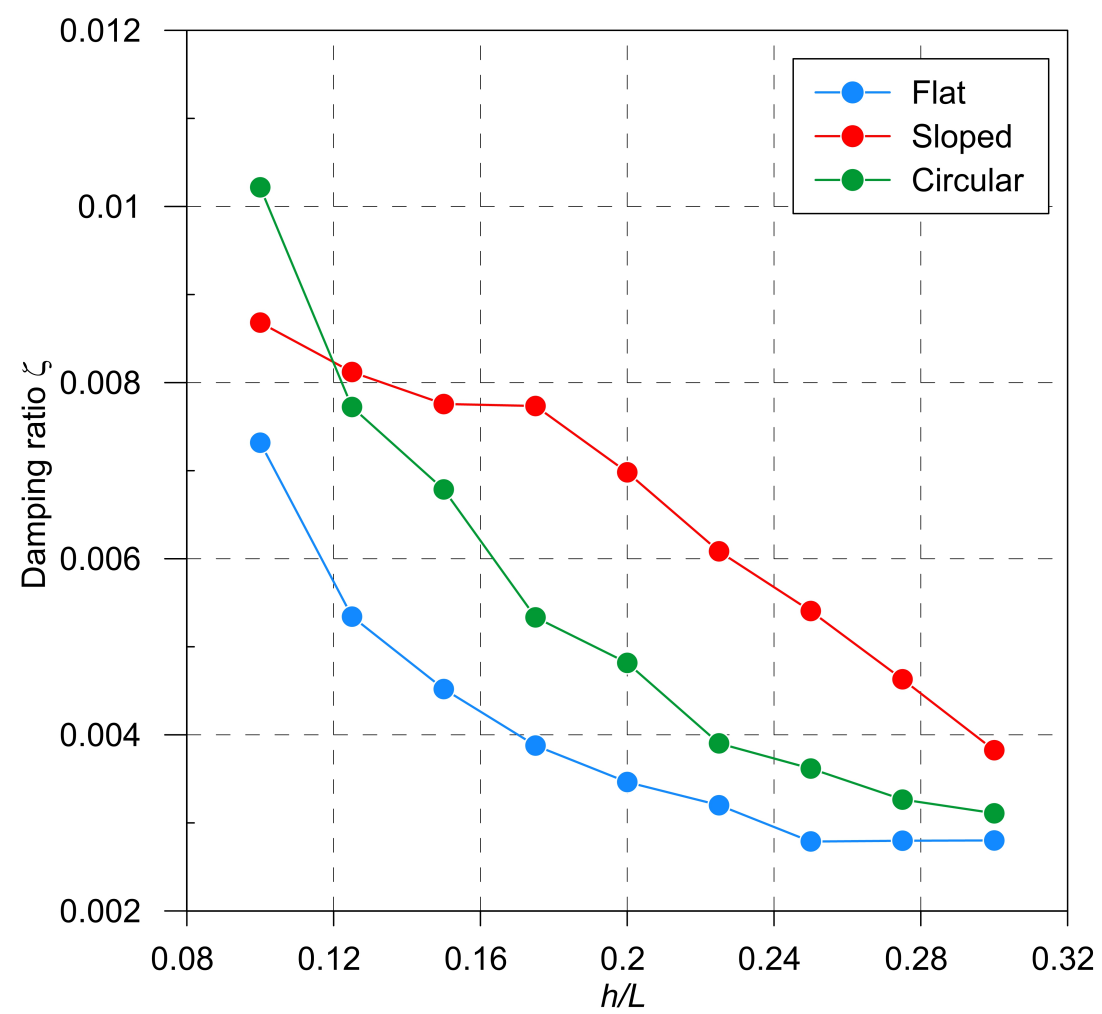

Figure 11. Damping coefficients vs. water depth ratio for different bottom geometries. 
As discussed above, the behaviour of the sloped bottom geometry from the first value of $h / L$ to $h / L=0.175$ initially increases while once the water depth equals the height of the wedge, its trend bends to finally keep increasing. Indeed, one of the main difficulties in modelling such an interacting system is the ability to properly define an equivalent mass actively participating in the overall sloshing process, that is to say having active or passive effects on the oscillating motion in the fluid-structure interaction. Another fundamental insight from Figure 10 is again the overall behaviour of the sloped bottom tank. While the flat and circular cases follow a monotonic increasing trend, the sloped one appears to be almost constant until the water depth reaches $9 \mathrm{~cm}$. This is due to the fact that until the water motion is affected by the sloped shape of the bottom side, the sloshing response remains substantially steady. In this sense, the sloped-bottom behaves like a softening spring because of the nonlinear effect of the wave run-up onto the sloping surface. This behaviour plays a crucial role in assessing that the use of slope shaped wedges guarantees a wider range of force impulses, that is to say a wider possibility to find constant tuning. Such a geometrical solution for the bottom of the tank results in the best option for a damping sloshing device.

Evidence of the above description can be found in the fundamental damping effectiveness related to the application of the three different bottom geometries. The damping mechanism depends on the amplitude of fluid sloshing and wave breaking patterns. The main driving mechanism of damping is attributable to viscous actions along the boundary layer near the bottom surface and the side walls of the tank and the sloshing motion of the free surface layer of the water, from which comes the importance of testing the behaviour under different bottom geometries. One of the issues, mainly in terms of proper modelling, is that a damper, deep water is not favourable because a large portion of the water does not participate in the damping mechanism.

The damping ratio, $\zeta$, is plotted in Figure 11 against water depth ratios. The following peculiar aspects can be drawn:

- the flat bottom shaped tank base is the least dissipative geometry, while the sloped one is the most;

- the circular bottom geometry is the most sensitive to the variation of the volume (mass) of water;

- $\quad$ only the sloped bottom seems to show a slightly concave trend with respect to the $x$-axis.

The first result was expected as anticipated and widely reported in literature [16,20,33]. The circular shape facilitates the liquid motion and, as a consequence, the mass of water interferes with the optimal tuning, depending on the liquid depth, producing alternatively a higher or lower dissipation. One interesting point concerns the fact that the flat and circular shapes exhibit a convex, flattening behaviour with the increasing water depth, whilst this is not observed for the sloped one. In terms of damping potential, the sloped bottom has a stronger sensitiveness to liquid mass, showing that this type of bottom geometry might affect the motion of the mass of water even at heights greater than the wedge vertical dimension. These considerations lead to the evidence that the sloped geometry might appear to have a contrasting behaviour: it has a more dissipative behaviour but it also shows a reaction delay with respect to the driven impulsive force. This "relaxation" in time can be seen as a consequence of the beating phenomenon that, in turn, can give an actual measure of the responsiveness of the overall system of liquid mass + structure.

\section{Conclusions}

In this paper the results of an experimental study on the characteristics of the free motion of water in prismatic sloshing devices with different bottom shapes have been presented. The performed experimental campaign consisted of a series of tests on a tank filled with water at nine different depths and each case tested for three different bottom geometries, namely flat, sloped and circular. The tank was subject to an impulsive random force and data about the related displacement and the dynamic inertia force have been 
acquired and processed in order to characterise the oscillating motion of the filling liquid and to focus on the so called "beating phenomenon". Since the interest was in the natural sloshing frequency and damping features, including the beating phenomenon, the sole measurement of the base shear force was deemed sufficient for the purpose of the work. Thence, further understanding of the free water surface dynamics considering different bottom geometries is left for future work.

Mechanical models of tanks filled with water in motion resemble the classical massspring-damper system and from this point of view equations to obtain natural frequency values have been formulated. An important objective of this work was to compare the equations outcomes with the experimental results and evaluate the effectiveness of such theoretical approaches to actual laboratory cases, even in the circumstance of aspect ratios $(h / L)$ higher than those allowed by the linear wave theory and in order to assess the validity of classical methods for practical tuning procedures. Furthermore, damping behaviour has been investigated considering the related aspect of the beating phenomenon.

Experimental natural frequency values for the box-shaped tank have shown a very good agreement with the corresponding values from Lamb's linear wave theory with a percentage error always under $2 \%$ for all the water depth ratios tested. The relative error was higher when compared with Deng's formulation because of the different theoretical approach which was more affected by the variation of liquid mass.

For the other two bottom shapes, namely sloped at $45^{\circ}$ and circular, the best analytical approach resulted to be the one proposed by Idir that, starting from the linear wave theory of Lamb and simplifying the wave dynamics equation with a small angles hypothesis, retrieved a modified expression for the natural sloshing frequency employing the wet perimeter. A further modification was to adapt the wet perimeter defined solely for wedged corners also for the case of the circular one.

The sloped shaped bottom showed a steady behaviour throughout all the nine different liquid depths with a slight increase of the oscillating frequency only for water heights over $9 \mathrm{~cm}$. This result is notable as it demonstrates that the sloped shape is the most robust with respect to the frequency "mistuning" that may be caused by varying water levels as it may happen in water storage tanks used for passive structural control purposes.

Author Contributions: Conceptualisation, A.A., N.C., C.B. and F.U.; methodology, N.C.; formal analysis, A.A.; investigation, N.C. and A.A.; data curation, A.A.; writing-original draft preparation, A.A.; writing-review and editing, A.A., N.C., C.B. and F.U.; supervision, C.B. and F.U.; project administration, C.B.; funding acquisition, C.B. All authors have read and agreed to the published version of the manuscript.

Funding: This research was supported by the Italian Ministry of Education, University and Research (MIUR) through the funded Project of Relevant National Interest "Combined numerical and experimental methodology for fluid structure interaction in free surface flows under impulsive loading" (Prot. 20154EHYW9).

Institutional Review Board Statement: Not applicable.

Informed Consent Statement: Not applicable.

Data Availability Statement: Not applicable.

Conflicts of Interest: The authors declare no conflict of interest.

\section{References}

1. Yalla, S.; Kareem, A. Beat phenomenon in combined structure-liquid damper systems. Eng. Struct. 2001, 23, 622-630. [CrossRef]

2. Breccolotti, M.; Gusella, V.; Materazzi, A. Active displacement control of a wind-exposed mast. Struct. Control. Health Monit. 2007, 14, 556-575. [CrossRef]

3. Materazzi, A.; Ubertini, F. Robust structural control with system constraints. Struct. Control. Health Monit. 2012, 19, 472-490. [CrossRef]

4. Venanzi, I.; Ierimonti, L.; Ubertini, F. Effects of control-structure interaction in active mass driver systems with electric torsional servomotor for seismic applications. Bull. Earthq. Eng. 2017, 15, 1543-1557. [CrossRef]

5. Faltinsen, O.M. A nonlinear theory of sloshing in rectangular tanks. J. Ship Res. 1974, 18, 224-241. 
6. Faltinsen, O.M. A numerical nonlinear method of sloshing in tanks with two-dimensional flow. J. Ship Res. 1978, 22, 193-202.

7. Armenio, V.; La Rocca, M. On the analysis of sloshing of water in rectangular containers: Numerical study and experimental validation. Ocean Eng. 1996, 23, 705-739. [CrossRef]

8. Ibrahim, R.; Pilipchuk, V.; Ikeda, T. Recent advances in liquid sloshing dynamics. Appl. Mech. Rev. 2001, 54, 133-199. [CrossRef]

9. Faltinsen, O.; Timokha, A. Natural sloshing frequencies and modes in a rectangular tank with a slat-type screen. J. Sound Vib. 2011, 330, 1490-1503. [CrossRef]

10. Herczyński, A.; Weidman, P. Experiments on the periodic oscillation of free containers driven by liquid sloshing. J. Fluid Mech. 2012, 693, 216-242. [CrossRef]

11. Cotana, F.; Belardi, P.; Manciola, P.; Tamagnini, C.; Materazzi, A.; Fornaciari, M.; Petrozzi, A.; Pisello, A.; Cavalaglio, G.; Coccia, V.; et al. TIAR: Renewable energy production, storage and distribution; a new multidisciplinary approach for the design of rural facility. Energy Procedia 2014, 45, 323-332. [CrossRef]

12. Cavalagli, N.; Biscarini, C.; Facci, A.; Ubertini, F.; Ubertini, S. Experimental and numerical analysis of energy dissipation in a sloshing absorber. J. Fluids Struct. 2017, 68, 466-481. [CrossRef]

13. Li, J.C.; Lin, H.; Li, K.; Zhao, J.F.; Hu, W.R. Dynamic Behavior in a Storage Tank in Reduced Gravity Using Dynamic Contact Angle Method. Microgravity Sci. Technol. 2020, 32, 1039-1048. [CrossRef]

14. Cooker, M.J. Water waves in a suspended container. Wave Motion 1994, 20, 385-395. [CrossRef]

15. Yu, J.K.; Wakahara, T.; Reed, D.A. A non-linear numerical model of the tuned liquid damper. Earthq. Eng. Struct. Dyn. 1999, 28, 671-686. [CrossRef]

16. Ibrahim, R.A. Liquid Sloshing Dynamics: Theory and Applications; Cambridge University Press: New York, NY, USA, 2005.

17. Sun, L.; Fujino, Y.; Pacheco, B.; Chaiseri, P. Modelling of tuned liquid damper (TLD). J. Wind Eng. Ind. Aerod. 1992, 43, 1883-1894. [CrossRef]

18. Tamura, Y.; Fujii, K.; Ohtsuki, T.; Wakahara, T.; Kohsaka, R. Effectiveness of tuned liquid dampers under wind excitation. Eng. Struct. 1995, 17, 609-621. [CrossRef]

19. Reed, D.; Yu, J.; Yeh, H.; Gardarsson, S. Investigation of tuned liquid dampers under large amplitude excitation. J. Eng. Mech. 1998, 124, 405-413. [CrossRef]

20. Tait, M.; El Damatty, A.; Isyumov, N. Testing of tuned liquid damper with screens and development of equivalent TMD model. Wind. Struct. 2004, 7, 215-234. [CrossRef]

21. Roy, A.; Zhang, Z.; Ghosh, A.; Basu, B. On the nonlinear performance of a tuned sloshing damper under small amplitude excitation. JVC J. Vib. Control 2019, 25, 2695-2705. [CrossRef]

22. Jing, W.; Feng, H.; Cheng, X. Dynamic Responses of Liquid Storage Tanks Caused by Wind and Earthquake in Special Environment. Appl. Sci. 2019, 9, 2376. [CrossRef]

23. Dou, P.; Xue, M.A.; Zheng, J.; Zhang, C.; Qian, L. Numerical and experimental study of tuned liquid damper effects on suppressing nonlinear vibration of elastic supporting structural platform. Nonlinear Dyn. 2020, 99, 2675-2691. [CrossRef]

24. Sun, L. Semi Analytical Modeling of Tuned Liquid Damper (TLD) with Emphasis on Damping of Liquid Sloshing. Ph.D. Thesis, University of Tokyo, Tokyo, Japan, 1991.

25. Russo, S.; Biscarini, C.; Facci, A.L.; Falcucci, G.; Jannelli, E.; Ubertini, S. Experimental assessment of buoyant cylinder impacts through high-speed image acquisition. J. Mar. Sci. Technol. 2018, 23, 67-80. [CrossRef]

26. Facci, A.L.; Falcucci, G.; Agresta, A.; Biscarini, C.; Jannelli, E.; Ubertini, S. Fluid Structure Interaction of Buoyant Bodies with Free Surface Flows: Computational Modelling and Experimental Validation. Water 2019, 11, 1048. [CrossRef]

27. Gabl, R.; Steynor, J.; Forehand, D.I.M.; Davey, T.; Bruce, T.; Ingram, D.M. Capturing the Motion of the Free Surface of a Fluid Stored within a Floating Structure. Water 2019, 11, 50 [CrossRef]

28. Den Hartog, J. Mechanical Vibrations, 4th ed.; McGraw-Hill: New York, NY, USA, 1957.

29. Cao, L.; Gong, Y.; Ubertini, F.; Wu, H.; Chen, A.; Laflamme, S. Development and validation of a nonlinear dynamic model for tuned liquid multiple columns dampers. J. Sound Vib. 2020, 487, 115624. [CrossRef]

30. Gurusamy, S.; Kumar, D. Experimental study on nonlinear sloshing frequency in shallow water tanks under the effects of excitation amplitude and dispersion parameter. Ocean Eng. 2020, 213, 107761. [CrossRef]

31. Phan Viet, N.; Kitano, Y.; Matsumoto, Y. Experimental Investigations of the Strengthening Effects of CFRP for Thin-Walled Storage Tanks under Dynamic Loads. Appl. Sci. 2020, 10, 2521. [CrossRef]

32. Konar, T.; Ghosh, A. Flow Damping Devices in Tuned Liquid Damper for Structural Vibration Control: A Review. Arch. Comput. Methods Eng. 2020. [CrossRef]

33. Gardarsson, S.; Yeh, H.; Reed, D. Behavior of sloped-bottom tuned liquid dampers. J. Eng. Mech. 2001, 127, 266-271. [CrossRef]

34. Olson, D.E.; Reed, D. A nonlinear numerical model for sloped-bottom tuned liquid dampers. Earthq. Eng. Struct. Dyn. 2001, 30, 731-743. [CrossRef]

35. Patil, G.; Singh, K. Evaluation of Sloped Bottom Tuned Liquid Damper for Reduction of Seismic Response of Tall Buildings. J. Inst. Eng. (India) Ser. A 2016, 97, 385-394. [CrossRef]

36. Bhosale, A.; Murudi, M. Seismic control of structures using sloped bottom tuned liquid dampers. Struct. Eng. Mech. 2017, 64, 233-241.

37. Pandit, A.; Biswal, K. Seismic control of multi degree of freedom structure outfitted with sloped bottom tuned liquid damper. Structures 2020, 25, 229-240. [CrossRef] 
38. Zhang, Z. Numerical and experimental investigations of the sloshing modal properties of sloped-bottom tuned liquid dampers for structural vibration control. Eng. Struct. 2020, 204. [CrossRef]

39. Deng, X.; Tait, M. Theoretical modeling of TLD with different tank geometries using linear long wave theory. J. Vib. Acoust. 2009, 131. [CrossRef]

40. Li, Y.; Wang, Z. An approximate analytical solution of sloshing frequencies for a liquid in various shape aqueducts. Shock Vib. 2014, 2014. [CrossRef]

41. Love, J.; Tait, M. Equivalent mechanical model for tuned liquid damper of complex tank geometry coupled to a 2D structure. Struct. Control. Health Monit. 2014, 21, 43-60. [CrossRef]

42. Love, J.; Tait, M. Linearized sloshing model for 2D tuned liquid dampers with modified bottom geometries. Can. J. Civ. Eng. 2014, 41, 106-117. [CrossRef]

43. Love, J.; Tait, M. The response of structures equipped with tuned liquid dampers of complex geometry. JVC J. Vib. Control 2015, 21, 1171-1187. [CrossRef]

44. Le Mehaute, B. An Introduction to Hydrodynamics and Water Waves; Springer: Berlin/Heidelberg, Germany, 1976; pp. 197-211.

45. Lamb, H. Hydrodynamics; Cambridge University Press: Cambridge, UK, 1916.

46. Idir, M.; Ding, X.; Lou, M.; Chen, G. Fundamental frequency of water sloshing waves in a sloped-bottom tank as tuned liquid damper. In Proceedings of the 2009 Structures Congress-Don't Mess with Structural Engineers: Expanding Our Role, Austin, TX, USA, 30 April-2 May 2009; pp. 831-840.

47. Bauer, H.F. Liquid oscillations with a free surface in wedge-shaped tanks. Acta Mech. 1981, 38, 31-54. [CrossRef] 